\title{
Effect of Physical Exercise on Cardio Metabolic Risk in Mexican Adolescents with Metabolic Syndrome
}

\author{
Oscar D Guzmán-Arredondo, Hector A Rubio-Zapata ${ }^{2 *}$, Carolina E Medina-Escobedo ${ }^{3}$ and \\ Damaris F Estrella-Castillo ${ }^{4}$
}

${ }^{1}$ Family Medicine Clinic, Division Yucatan, Mexican Social Security Institute, Mexico

${ }^{2}$ Department of Clinical and Epidemiological Research, Autonomous University of Yucatan, Mexico

${ }^{3}$ Yucatan High Specialty Medical Hospital, Mexican Institute of Social Security, Mexico

${ }^{4}$ Rehabilitation Department, Autonomous University of Yucatan, Mexico

*Corresponding author: Hector A Rubio-Zapata, Department of Clinical and Epidemiological Research, Medicine School, Autonomous University of Yucatan, Itzaez Avenue 498, downtown, Mérida, Yucatán, México

\section{ARTICLE INFO \\ Received: 幽 August 23, 2021 \\ Published: 幽 September 08, 2021}

Citation: Oscar D Guzmán-Arredondo, Hector A Rubio-Zapata, Carolina E Medina-Escobedo, Damaris F EstrellaCastillo. Effect of Physical Exercise on Cardio Metabolic Risk in Mexican Adolescents with Metabolic Syndrome. Biomed J Sci \& Tech Res 38(4)-2021. BJSTR. MS.ID.006185.

Keywords: Exercise; Insulin Resistance; Insulin Sensitivity; Metabolic Syndrome; Adolescent

\begin{abstract}
Cardiovascular and metabolic pathologies are the first causes of mortality in Mexico. Cardio metabolic risk is greater in adolescents with metabolic syndrome (MetS). Physical exercise has proven to be a therapeutic tool in the management of MetS. The objective was to analyze the effect that moderate intensity physical exercise for 12 weeks has on components of MetS and cardiometabolic risk in adolescents in southeastern Mexico. Methodology. Quasi-experimental design. Thirty-six adolescents with MetS performed aerobic exercise for 12 weeks. Was determined at the beginning and end of the intervention: abdominal obesity, hypertension, hyperglycemia, hypertriglyceridemia, hypo-alpha-lipoproteinemia, insulin resistance, insulin insensitivity, B-pancreatic cell function, atherogenic risk, excess weight, and excess body fat, among other risks. Data were analyzed with t-Student, square chi and bivariate analysis. Results, exercise significantly lowered systolic and diastolic blood pressure $(\mathrm{P}<0.001)$, triglycerides $(\mathrm{P}=0.01)$ and insulin resistance $(\mathrm{P}=0.007)$. Increased $\mathrm{B}$-pancreatic cell function $(\mathrm{P}=0.02)$. At the end of intervention 32 adolescents (84\%) recovered from MetS. Conclusion, physical exercise reverses MetS on Mexican adolescents and reduces cardiometabolic risk.
\end{abstract}

\begin{abstract}
Abbreviations: MetS: Metabolic Syndrome; IR: Insulin resistance; HOMA-S: Insulin Insensitivity; HOMA-B: B-Pancreatic Cell Function; AR: Atherogenic Risk; EW: Excess weight; EBF: Excess Body Fat; WC: waist circumference; SBP: systolic blood pressure; IDF: International Diabetes Federation; DBP: Diastolic Blood Pressure; MAP: mean arterial pressure
\end{abstract}

\section{Introduction}

The metabolic syndrome (MetS) is a set of dysfunctions and disorders that increase the risk of developing chronic diseases mainly of cardiovascular and metabolic type, such as diabetes mellitus, coronary disease, stroke and others that currently represent the main causes of morbimortality in Mexico [1]. Although MetS is more prevalent in the adult population, recently an increase in its incidence has been observed in children and adolescents, which is worrisome due to the health risks this represents. Some authors believe that increase in the incidence of MetS in children and adolescents is due to the high prevalence of obesity, excessive caloric intake and sedentary lifestyles [2]. At world level, Mexico occupies the first places of obesity in pediatric population, therefore it is estimated MetS represent a public health problem in children and adolescents [3]. The diagnosis of MetS 
in adolescents includes elevated blood pressure levels, increased waist circumference, increased triglyceride levels and fasting glycaemia, as well as decreased HDL cholesterol [4]. There are several guidelines for diagnosis MetS in adolescents. The diagnosis must be adjusted with age and sex [5], since adolescence is a period of rapid growth, in addition to the dimorphic effect of sex hormones on fat tissue [6]. Cardiometabolic risk is the likelihood that a person will have cardiovascular damage, it can be determined with different mathematical models based on the numbers of certain metabolites such as insulin, lipid profile, glycemia and others.

Among the main indicators of cardiometabolic risk are: insulin resistance or insensitivity, pancreatic beta cell dysfunction and atherogenic risk, which represent a poor prognosis for health $[7,8]$. MetS can be treated with different therapeutic approaches, such as lifestyle modification, pharmacological treatments and even surgical interventions [9]. Physical exercise is one of the strategies that have been successfully used to manage MetS and some other metabolic diseases such as diabetes mellitus and obesity [10]. Some publications have reported that exercise reverses MetS [11,12] having therapeutic effects on its components, however others have found no effects on MetS, but they report benefits in other metabolites such as adipokines and other inflammatory markers $[13,14]$. This controversy may be related to the type and duration of exercise. Physical exercise is an accessible activity for most people, it is economic, and it can also have a recreational component, which tends to be attractive to adolescents and to improve adherence to treatment, which is one of the keys to therapeutic success [15]. In Mexico, some studies have estimated that the highest prevalence of MetS in adolescents is found in the southeast of the country, probably due to the high rates of obesity and overweight in this same region $[16,17]$ and make it necessary to implement therapeutic strategies to improve health in this population group.

The goal of the present study was to analyze the effect that moderate intensity physical exercise for 12 weeks has on components of MetS and cardiometabolic risk in adolescents in southeastern Mexico.

\section{Methodology}

Quasi-experimental study was conducted on adolescents with MetS in Merida, Yucatan, Mexico. MetS components and cardiometabolic risks were analyzed before and after physical exercise model. Sample selection. Adolescents aged 15 to 18 registered in high schools were invited to participate in the study. Was a non-probabilistic sample of 313 adolescents that accepted to participate through the letters of assent and informed consent. After first evaluation, 46 met MetS criteria of which 86\% complete de exercise model. MetS diagnosis. Based on the criteria of the Latin American Diabetes Association (4) (ALAD), which considers presenting at least three of the five indicators proposed by the
International Diabetes Federation (IDF) adapted to the adolescent population: Abnormal fasting glycemia (100-126 mg/dl), hypolipoproteinemia [cholesterol-HDL $\leq 40 \mathrm{mg} / \mathrm{dl}$ (men) and $\leq 50$ $\mathrm{mg} / \mathrm{dl}$ (women)], hypertriglyceridemia ( $\geq 150 \mathrm{mg} / \mathrm{dl}$ ), hypertension [systolic blood pressure (SBP) and /or diastolic blood pressure (DBP) $\geq$ percentile 95] and abdominal obesity [waist circumference (WC) $\geq$ percentile 90]. Anthropometric and clinical measurements. Carried out with fasting of 10-12 hours. Weight (Kg) and body fat percentage was determined with a TANITA Onrom ${ }^{\circledR}$ TBF 300GS body composition scale. Size was determined with the SECA $^{\oplus}$ stadiometer. Body max index (BMI), was established by the formula height $\left(\mathrm{m}^{2}\right) /$ weight $(\mathrm{Kg})$.

We classified as excess weight those values equal to or greater than the $85^{\text {th }}$ percentile that include overweight and obesity, according to the Official Mexican Standard NOM-047-SSA2-2015 [18]. Body fat was classified as normal or excess, considering age and sex, according to the cut-off points for pediatric patients mentioned by McCarthy et al. [19] Waist circumference was determined with $\mathrm{SECA}^{\circledast}$ brand tape at the navel level at the end of a normal exhalation and was expressed in centimeters [4]. Blood pressure was measured with an Omron ${ }^{\circledR}$ brand HEM-7200 digital baumanometer, hypertension was diagnosed when SBP or DPB figures $\geq 95^{\text {th }}$ percentile, in three different measurements. The mean arterial pressure (MBP) was calculated according to the formula SBP-DBP/3+DBP [20]. Biochemical analysis. Performed on venous blood samples, after 10-12 hours of fasting. Glucose and lipid profile measurements were performed in the automated Dimension Chemistry System, Dade Behring ${ }^{\circledR}$. Serum insulin was determined by immunoadsorption techniques in the Beckman Coulter $^{\circledR}$ equipment, exposed in $\mu \mathrm{U} / \mathrm{ml}$. Hyperinsulinaemia was established with values of $23 \mu \mathrm{U} / \mathrm{ml}$ or $>$ according to the reference standards of the clinical analysis laboratory. The handling of biological residues was in accordance with the Mexican Technical Norm NOM-087-ECOL-SSA1-2002.

Cardiometabolic Risks Indexes. HOMA-IR Index (homeostasis model assessment). Fasting plasma insulin value $(\mu \mathrm{U} / \mathrm{mL}) \mathrm{x}$ fasting plasma glucose $(\mathrm{mg} / \mathrm{dL}) / 22.5$. HOMA-RI in men $>2.29$ and in women > 2.21 [21]. QUICKI (quantitative insulin sensitivity check index) is a variation of the HOMA equation, which transforms the data by taking the logarithm and the reciprocal of the glucose-insulin product, according to the formula [1/ (log insulin + log glucose)]. The values used for RI QUICKI were $0.382 \pm 0.007$ for non-obese and $0.331 \pm 0.010$ for obese (7) HOMA Index B. Represents the HOMA of the pancreatic beta cell function and was determined with the following formula: 20 [fasting insulin $(\mu \mathrm{U} / \mathrm{ml}) /$ fasting glucose (mmol/ml)-3.5]. The cut-off point of decreased beta cell function was less than $150 \%$ [22]. HOMA-S Index. Represents the HOMA model of insulin sensitivity and is used to provide information about glucose metabolism, this value was calculated as 1 HOMA- 
IR $x$ 100. Values $>54.1$ for women and $>46.1$ for men indicated insulin insensitivity [23]. Triglyceride/glucose index is an indicator of insulin resistance which was determined with the natural log of triglycerides $(\mathrm{mg} / \mathrm{dL}) \times$ glucose $(\mathrm{mg} / \mathrm{dL}) / 2$. The criteria used was 8.8 for men and 8.7 for women [8] Indicator triglycerides/HDL-c was calculated with the ratio triglycerides (mg/dL)/HDL-c(mg/ $\mathrm{dL}$ ), the limit value was 3.1 for men and 2.2 for women (8). Plasma atherogenic index was calculated using the $\log (\mathrm{TG} / \mathrm{HDL}-\mathrm{c})$ formula. Values 0.11 or $>$ were considered to be at risk [24]. Cardiovascular atherogenic index was determined with the HOMA-IR $x$ TG/HDLc formula. Value $>28$ is considered to be at risk.

Exercise model. Consisted of moderate intensity aerobic exercise (walking, running, jumping and stretching) three times a week for 3 months. The sessions were held outdoors in the morning, lasting 30 minutes. All practices were indicated and supervised. Only 36 participants completed the exercise model. The study was conducted in accordance with the ethical provisions and recommendations issued in the Declaration of Helsinki of the World Medical Association, as well as the Regulations on Research of the General Health Law of Mexico NOM-012-SSA3-2012. The protocol was approved by the Local Committee of Ethics and Research of the Mexican Institute of Social Security (IMSS), registration R-20183203-038.

\section{Statistics}

The statistical analysis included averages, standard deviations, medians and ranges. The normality of the variables was established with Shapiro-Wilk test. The components of the MetS and the cardiometabolic risk indexes were expressed as percentages. The difference $(\Delta)$ between the initial and final quantitative measurements of each group determined. t-Student tests were used for compared normal data. U-Mann Whitney test were used in non-normal data. Analysis of nominal data were performed with chi-square tests or Fisher's exact test. SPSS Version 23 software was used (IBM Corp., Armonk, NY, USA). The P-Values $\leq .05$ were considered statistically significant.

\section{Results}

MetS prevalence on the population was $14.69 \%$, with average age of $15.4 \pm .6$ years. (Table 1 ) describes the initial and final values of the clinical, anthropometric and laboratory measurements, and it's comparison. Physical exercise reduced all blood pressure indicators (SBP, DBP and MBP) and triglyceride levels as shown in (Table 1). Based on the cut-off points of the different measurements mentioned in the methodology, the components of MetS, some metabolic dysfunctions and the cardiometabolic risks indexes was determined. The percentages and variation $(\Delta)$ between initial and final measures are expressed in (Table 2). According to the order of frequency, the profile of MetS on Mexican adolescent was hypoaliproteinemia, abdominal obesity, hypertension, hypertriglyceridemia, and abnormal fasting glycemia; their percentages are shown in (Table 2). Physical exercise model reduced arterial hypertension (SBP and DBP) and increased insulin sensitivity and pancreatic beta function. Median of MetS components initially was tree and at the end of the intervention was zero ( $\mathrm{p}<0.0001$, Wilcoxon, data no shown), and 34 adolescents $(84 \%)$ recovered from MetS.

Table 1: Anthropometric, clinical, and laboratory values of Mexican adolescents with MetS, after and before 12 weeks physical exercise.

\begin{tabular}{|c|c|c|c|}
\hline & Initial & Final & P* \\
\hline Weight (Kg) & $79.7 \pm 15.6$ & $80.3 \pm 15.5$ & .32 \\
\hline Size (m) & $1.60 \pm .07$ & $1.60 \pm 1$ & .77 \\
\hline BMI & $30.9 \pm 5.6$ & $30.9 \pm 5.3$ & .90 \\
\hline Waist (cm) & $100 \pm 12.2$ & $98.7 \pm 13.7$ & .36 \\
\hline SBP (mm/Hg) & $125.5 \pm 9.3$ & $109.7 \pm 12.7$ & $<.001$ \\
\hline DBP (mm/Hg) & $78.8 \pm 8.4$ & $68.8 \pm 6.5$ & .001 \\
\hline MBP (mm/Hg) & $91.1 \pm 5.8$ & $82.4 \pm 7$ & $<.001$ \\
\hline Glucose (mg/dl) & $87.4 \pm 5.7$ & $84.3 \pm 5.5$ & .08 \\
\hline Fat (\%) & $37.6 \pm 8.3$ & $36.4 \pm 7.9$ & .18 \\
\hline Total Cholesterol (mg/dl) & $145.7 \pm 34.4$ & $153.2 \pm 25.6$ & .29 \\
\hline Trig (mg/dl) & $135.4 \pm .75$ & $103.1 \pm 39.9$ & .01 \\
\hline HDL-c (mg/dl) & $40.6 \pm 7.9$ & $42 \pm 6.6$ & .28 \\
\hline Insulin $(\mathrm{uU} / \mathrm{dl})$ & $14.8(5.1-79)$ & $13.4(7.1-45.8)$ & .55 \\
\hline
\end{tabular}

Note: MetS, metabolic syndrome; \%, percentage; cm, centimeter; BMI, body mass index; mm/Hg, millimeters of mercury; SBP, systolic blood pressure, DBP, diastolic blood pressure; MBP, mean blood pressure. Col, Cholesterol; Trig, Triglycerides; HDL-c, High Density Cholesterol; mean \pm standard deviation; † median (minimum-maximum). $\mathrm{t}-$ Student related samples, $† \mathrm{U}$ Mann-Whitney 
Table 2: Cardio-metabolic risk and other dysfunctions in Mexican adolescents with MetS, before and after 12 weeks physical exercise.

\begin{tabular}{|c|c|c|c|c|}
\hline Variable & Initial & Final & $\mathbf{P}^{*}$ & $\Delta$ \\
\hline Abdominal Obesity & $63 \%$ & $68 \%$ & .74 & $5 \%$ \\
\hline SAH & $42 \%$ & $10 \%$ & .02 & $-32 \%$ \\
\hline DAH & $42 \%$ & $5 \%$ & .008 & $-37 \%$ \\
\hline Abnormal fasting glycemia & $5 \%$ & $0 \%$ & .33 & $-5 \%$ \\
\hline Hypertriglyceridemia & $36 \%$ & $15 \%$ & .14 & $-21 \%$ \\
\hline Hypoalphalipoproteinemia & $80 \%$ & $68 \%$ & .40 & $-12 \%$ \\
\hline Excess Weight & $15 \%$ & $15 \%$ & 1 & $0 \%$ \\
\hline Excess Body Fat & $94 \%$ & $79 \%$ & .18 & $-15 \%$ \\
\hline Hyperinsulinemia & $15 \%$ & $21 \%$ & .63 & $6 \%$ \\
\hline HOMA IR & $89 \%$ & $78 \%$ & .36 & $-11 \%$ \\
\hline IR QUICKI & $68 \%$ & $47 \%$ & .19 & $-21 \%$ \\
\hline HOMA 1-S & $100 \%$ & $68 \%$ & .007 & $-32 \%$ \\
\hline HOMA Beta & $10 \%$ & $42 \%$ & .02 & $32 \%$ \\
\hline IR Tg/Gluc & $37 \%$ & $15 \%$ & .12 & $-22 \%$ \\
\hline IR Tg/HDL-c & $57 \%$ & $36 \%$ & .20 & $-21 \%$ \\
\hline IAC & $10 \%$ & $5 \%$ & .56 & $-5 \%$ \\
\hline AIP & $94 \%$ & $80 \%$ & .20 & $-14 \%$ \\
\hline
\end{tabular}

Note: MetS Metabolic syndrome. SAH, systolic arterial hypertension, DAH, diastolic arterial hypertension; $\%=$ percentage; $\Delta$ percentage of variation= initial value-final value; HOMA-IR, mathematical model of insulin resistance; QUICKI, quantitative index of insulin sensitivity; HOMA 1-S, index of insulin sensitivity; trig, triglycerides; glucose; HDL-c, High density cholesterol; RI, insulin resistance; IAC, cardiovascular atherogenic index. AIP, plasma atherogenic index. Statistical, square chi or Fisher test.

\section{Discussion}

The present study focused on evaluating the effect of physical exercise in adolescents with MetS and their cardiometabolic risk. The reduced sample size facilitated exercise monitoring, practiced under the same conditions. We found a prevalence of MetS 15\% in adolescents between 15 and 18 years of age, following the diagnostic criteria of the ALAD. Ramirez, et al. $[25,26]$, point out that this classification is ideal for Mexican adolescents. It is very likely that using other criteria such as the IDF or the ATPIII, the prevalence is higher since the use more general MetS diagnostic criteria. The profile of MetS found in the Mexican adolescents was hyperalphalipoproteinemia, abdominal obesity, hypertension, hypertriglyceridemia and abnormal fasting glycemia. In young adults in Brazil, the profile of MetS was hypoalpha-lipoproteinemia, hypertriglyceridemia and hypertension; [27] in Peruvian adolescent's hyperalphalipoproteinemia, hypertriglyceridemia and abdominal obesity were found [28], both studies indicate that abnormal fasting glycemia was the least frequent component. We found similarities between these profiles, highlighting hyperalphalipoproteinemia as the most frequent component of MetS. Atherogenic dyslipidemia is made up of hypoalphalipoproteinemia and hypertriglyceridemia, which is worrisome because of its pathogenic implications. The frequency of dyslipidemias found by Sapunar, et al. [24] in Chilean adolescents was 38\% and by Pajuelo, et al. [29] in Peruvian adolescents with obesity and IR was $46 \%$.

We found $80 \%$ of hypoalphalipoproteinemia and $30 \%$ hypertriglyceridemia. Dyslipidemias are related to high fat diets, obesity and physical inactivity. [24] It is likely that the gastronomy southeast region of Mexico, being high in saturated and unsaturated fats, is a contributor to the high incidence of dyslipidemias. A limitation of the study was not to analyze the diet of the adolescents, although they were asked to maintain their usual diet to avoid interference with results. Genetics is a factor that can alter the metabolism of fats, differences have been found in the lipid profile between ethnic groups and races, which have been associated with genetic polymorphisms or epigenetic characteristics [29]. Gonzales, et al. [30] in children from this region found polymorphisms associated with obesity and overweight, which may be related to dyslipidemia and MetS. Another factor alters the lipid profile is IR, whose effect at the hepatic level is to increase the synthesis of very low-density lipoproteins, increase the release of low-density cholesterol lipoproteins and triglycerides in the blood, and can also decrease HDL-c by increasing the speed of its degradation and by the abnormal maturation of apolipoprotein 1/HDL-c [21]. Although the gold standard for IR is the euglycemic clamp, this tool was not accessible, so the IR was determined by insulin values. With these methods we found $68-89 \%$ of IR in adolescents with MetS. 
Using lipid values to determine the IR prevalence in adolescents with MetS was $37-57 \%$, which seems to be more reliable but less sensitive, and may be a more accessible alternative to identify IR in the first level of health care since serum insulin tends to be more expensive and less accessible [31]. Interestingly, during adolescence, which is a period of growth, somatotropin modifies glucose metabolism and produces compensatory hyperinsulinism. This occurs in stage IV and V adolescents according to the Tanner scale $[6,21]$ which corresponds by age to the adolescents studied. Excess body fat was found in $94 \%$ of adolescents with MetS. We found excess fat, hyperinsulinemia and insulin sensitivity, subclinical alterations that can favor the development of cardiometabolic diseases [32]. Some adolescents without abdominal obesity presented excess body fat, which may be indicating visceral obesity, some authors have called these people as TOFI (Thin Outside, Fat Inside), which is changing the paradigm of obesity and highlights the need for other tools to identify pathogenic adiposity, since fat affects the metabolism of lipids and carbohydrates and therefore may increase the frequency of MetS and cardiometabolic risk [33]. Exercise decreased blood pressure levels and triglyceride levels in adolescents with MetS. In accordance with these results, different studies have found that the main effect of exercise is the reduction of blood pressure; it seems that muscle activity improves circulation and favors the production of nitric oxide, produces vasodilation which lowers blood pressure.

Nitric oxide may also decrease oxidative stress and improve insulin function in the muscle cell, which represents protective effects at the cardiovascular level [34]. Other authors have also found that physical exercise reduces triglyceride levels in children and adolescents, proposing that the energy spent on exercise comes from the hydrolysis of blood triglycerides and muscle deposits [35]. Meta-analyses have concluded that exercise decreases body fat, both visceral and abdominal, especially when it is of high intensity [36]. Exercise increased beta-pancreatic cell function, this indicator must be correlated with insulin resistance to give a clinical prognosis [37]. In obese adolescents in Taiwan, an intervention similar to ours resulted in decreased body fat, increased insulin sensitivity, and improved b-pancreatic function [38]. The atherogenic index of plasma, a risk factor for coronary disease, was elevated in adolescents with MetS. This risk was high, probably because of diet. González, et al. [39] in European adolescents propose that a diet rich in saturated and hyperglycemic fats increase cardiovascular risk. Adolescence represents a key period where it is possible to adopt healthy behaviors and adolescents with MetS should be made aware of the importance of lifestyle, as well as encourages physical exercise as a habit that can improve cardiometabolic health and prevent the development of chronic diseases in adulthood.

\section{Conclusion}

In Mexican adolescents with MetS, physical exercise lowers blood pressure levels, triglycerides and improved some cardiovascular risk indicators such as insulin sensitivity and pancreatic beta cell function. We propose that by increasing the intervention time with physical exercise greater therapeutic effects would be achieved.

\section{Conflicts of Interest}

All the authors declare no conflict of interest.

\section{Acknowledgements}

We are grateful to the Autonomous University of Yucatan for all the facilities provided to carry out this work.

\section{References}

1. Morales VM, Jardón RA (2014) Risk Factors of Metabolic Syndrome in adolescents of 12-16 Years old. Med Int Mex 30(2): 152-158.

2. Magge SN, Goodman E, Armstrong SC (2017) The Metabolic Syndrome in Children and Adolescents: Shifting the Focus to Cardiometabolic Risk Factor Clustering. Pediatrics 140(2): e1-e14

3. Shamah LT, Cuevas NL, Gaona PB, Gomez AL, Morales RM, et al. (2018) Overweight and obesity in children and adolescents, 2016 Halfway National Health and Nutrition Survey update. Salud Publica Mex 60(3): 244-253.

4. Zimmet P, Alberti K, George MM, Kaufman F, Tajima N, et al. (2007) The metabolic syndrome in children and adolescents an IDF consensus report. Pediatric Diabetes 8(5): 299-306.

5. Ma C, Lu Q, Wang R, Yin F (2019) Using height-corrected definition of metabolic syndrome in children and adolescents. J Pediatric Endocrin Metab 32(5): 429-438.

6. Shin HK, Mi JP (2017) Effects of growth hormone on glucose metabolism and insulin resistance in human. Ann Pediatric Endocrin Metab 22(3): 145-152.

7. Gutch M Kumar S, Razi SM, Gupta KK, Gupta A (2015) Assessment of insulin sensitivity/resistance. Indian J Endocrin Metab 18(1): 160-164.

8. Unger G, Benozzi S, Perruzza F, Penacchiotti G (2014) Triglycerides and glucose index: A useful indicator of insulin resistance. Endocrin Nutr 61 (10): 533-540.

9. Guerreiro V, Neves JS, Salazar D, Ferreira MJ, Castro O, et al. (2019) LongTerm Weight Loss and Metabolic Syndrome Remission after Bariatric Surgery: The Effect of Sex, Age, Metabolic Parameters and Surgical Technique - A 4-Year Follow-Up Study. Obes facts 12: 639-652.

10. López J (2010) Hypertension treatment in patients with metabolic syndrome. Rev Colomb Cardiol 17(1): 22-27

11. Aoi W, Naito Y, Yoshikawa T (2011) Dietary exercise as a novel strategy for the prevention and treatment of metabolic syndrome: Effects on Skeletal Muscle Function. J Nutr Metabol 2011: 676208.

12. Gondim OS, Camargo VTNd, Gutierrez FA, Martins PFdO, Passos MEP, et al. (2015) Benefits of Regular Exercise on Inflammatory and Cardiovascular Risk Markers in Normal Weight, Overweight and Obese Adults. PLoS One 10(10): e0140596. 
13. Landeros O, López A, Nava G, Gallegos C, Lavalle G, et al. (2014) Effect of cardiovascular exercise on women with obesity on the concentrations of adiponectin, leptin and tumor necrosis factor-alpha. Arch Cardiol Mex 84(3): 177-182.

14. Mendham E, Duffield R, Marino F, Cuttts J (2015) A 12-week sportsbased exercise programme for inactive indigenous Australian men improved clinical risk factors associated with type 2 diabetes mellitus. J Sci Med Sport. 18 (4): 438-443.

15. Ruegsegger N, Booth W (2018) Health Benefits of Exercise. Cold Spring Harb Perspect Med 8(7).

16. Secretaria de Salud. Encuesta Nacional de Salud y nutricion (ENSANUT 2016).

17. Murguía R, Jiménez F, Villalobos M, Méndez C (2012) Estimating the geographical distribution of the prevalence of the metabolic syndrome in young Mexicans. Geospat Health 6(3): S43-S50.

18. Norma Oficial Mexicana NOM-047-SSA2-2015, Para la atención a la salud del Grupo Etario de 10 a 19 años de edad.

19. McCarthy HD, Cole TJ, Fry T, Jebb SA, Prentice AM (2006) Body fat reference curves for children. Int J Obes 30(4): 598-602.

20. Acoltzin V, Rabling A, Marcial G (2010) Diagnosis of hypertension based on the calculation of the average blood pressure. Rev Mex Cardiol 21(3): 99-103.

21. García C, García L, Jiménez L, González V, Calvo R, et al. (2007) Index HOMA y QUICKI, insulin and C-peptide in healthy children. Cardiovascular risk cut-off points. An pediatr (Barc) 66(5): 481-490.

22. Matthews D, Hosker P, Rudenski S, Naylor B, Treacher D, et al. (1985) Homeostasis model assessment: Insulin resistance and $\beta$-cell function from fasting plasma glucose and insulin concentrations in man. Diabetol 28(7): 412-419.

23. Ghasemi A, Tohidi M, Derakhshan A, Hasheminia M, Azizi F, et al. (2015) Cut-off points of homeostasis model assessment of insulin resistance, beta-cell function, and fasting serum insulin to identify future type 2 diabetes: Tehran Lipid and Glucose Study. Acta Diabetol 52 (5): 905-915.

24. Sapunar J, Aguilar F, Navarro J, Araneda G, Chandía P, et al. (2018) High prevalence of dyslipidemia and high atherogenic index of plasma in children and adolescents. Rev Med Chile 146(10): 1112-1122.

25. Bertoluci M, Quadros A, Sarmento L, Schaan B (2010) Insulin resistance and triglyceride/HDLc index are associated with coronary artery disease. Diabetol Metab Syndr 2: 11.

26. Ramírez D, Luna H (2019) Prevalencia de síndrome metabólico en niños y adolescentes mexicanos, en torno a sus diferentes definiciones. Rev Salud Publica Nutr 18(2): 23-32.

ISSN: 2574-1241

DOI: 10.26717/BJSTR.2021.38.006185

Hector A Rubio-Zapata. Biomed J Sci \& Tech Res

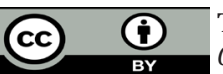

This work is licensed under Creative

Commons Attribution 4.0 License

Submission Link: https://biomedres.us/submit-manuscript.php
27. Vilarouca da Silva A, Nascimento da Sousa L, de Sousa R, Alves C, Goncalves M, et al. (2014) Prevalence of metabolic components in university students. Rev Lat Am Enfermagem 22(6): 1041-1047.

28. Ninanta O, Núñez Z, García F, Romaní R (2016) Frequency of metabolic syndrome in residents of an Andean region of Peru. Rev Peru Med Exp Salud Publica 33(4): 640-650.

29. Pajuelo R, Bernui L, Sánchez G, Arbañil H, Miranda C, et al. (2018) Obesidad, resistencia a la insulina y diabetes mellitus tipo 2 en adolescentes. An Fac med 79(3): 200-205.

30. González H, Zavala C, Ayala C, Pérez M, López G, et al. (2019) Genetic variation of FTO: rs1421085 T>C, rs8057044 G>A, rs9939609 T>A, and copy number (CNV) in Mexican Mayan school-aged children with obesity/overweight and with normal weight. Am J Hum Biol 31(1): e23192.

31. Locateli C, Arthur L, Ferraz S, de Olviera G, Oltramari K, et al. (2019) Triglyceride/glucose index is a reliable alternative marker for insulin resistance in South American overweight and obese children and adolescents. J Pediatr Endocrinol Metab 32(10): 1163-1170.

32. Cruz R, González V, Reyes C, Mayorga R, Najera M, et al. (2019) Food intake and body composition associated with metabolic syndrome in university students. Revista Mexicana de Trastornos Alimentarios 10(1): 42-52

33. Chaldakov G (2017) Obesity: An Inside Versus an Outside View. Jimmy Bell and The Little Prince a Science-In-Fiction Dedicated To World Obesity Day 2017. Scripta Scientifica Vox Studentium 1(1): 13-17.

34. Donley D, Fournier S, Reger B, DeVellance E, Bonner D, et al. (2014) Aerobic exercise training reduces arterial stiffness in metabolic syndrome. J Appl Physiol 116 (11): 1396-1404.

35. Pablos A, Nebot V, Vaño V, Ceca D, Elvira L (2018) Effectiveness of a school-based program focusing on diet and health habits taught through physical exercise. Appl Physiol Nutr Metab 43(4): 331-337.

36. Maillard F, Pereira B, Boisseau N (2018) Effect of High-Intensity Interval Training on Total, Abdominal and Visceral Fat Mass: A Meta-Analysis. Sports Med 48: 269-288.

37. Buccini G, Wolfhal D (2008) Cut-off values for indices of insulin resistance, sensitivity and secretion derived from the HOMA formula and HOMA2 program: Interpretation of data. Rev argent endocrinol metab 45(1): 3-21.

38. Shih KC, Kwok CF (2018) Exercise reduces body fat and improves insulin sensitivity and pancreatic $\beta$-cell function in overweight and obese male Taiwanese adolescents. BMC Pediatr 18(1): 18-80.

39. González G, Santabárbara J, Ruiz S, Bel S, Huybrechts I, et al. (2017) Ideal cardiovascular health and inflammation in European adolescents: The HELENA study. Nutr Metabol Cardio Dis 27(5): 447-445.

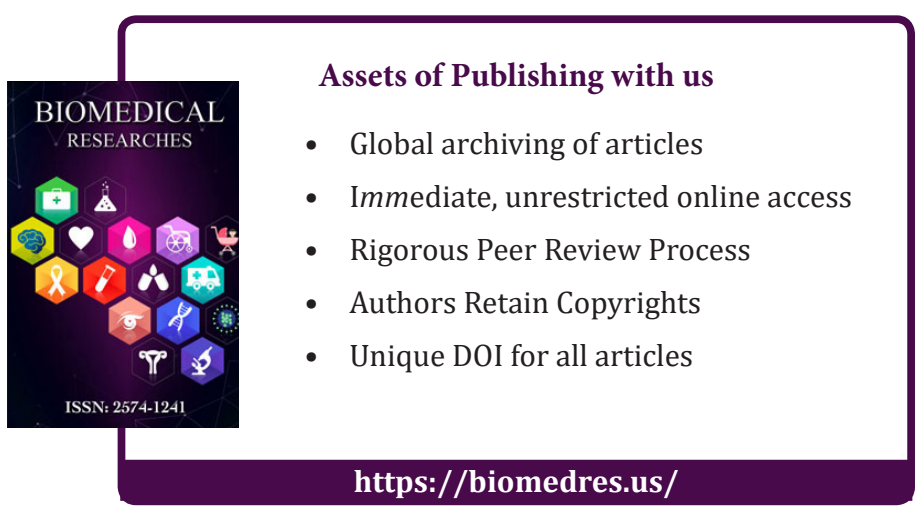

